\title{
Superconducting Metamaterials
}

\author{
Michael Ricci, Nathan Orloff and Steven M. Anlage \\ Center for Superconductivity, Physics Department, University of Maryland, College Park, MD 20742
}

Evanescent wave amplification has been predicted under the ideal condition that the index of refraction, $n=-1+i 0$ precisely, but is difficult to observe in practice because current metamaterials suffer from high losses. We present experimental results on a metamaterial that employs superconducting $\mathrm{Nb}$ metals and low-loss dielectric materials. Results include transmission data on a wire, split-ring resonator, and a combination medium at temperatures between $4.2 \mathrm{~K}$ and $297 \mathrm{~K}$. Evidence of negative effective permittivity, permeability, and a negative effective index passband are seen in the superconducting state between $50 \mathrm{MHz}$ and $18 \mathrm{GHz}$. We find a dielectric loss of $\varepsilon_{e f f, 2}$ $=2.6 \times 10^{-3}$ in a superconducting wire array at $10.75 \mathrm{GHz}$.

There has been renewed interest in the properties of materials with a negative index of refraction (NIR), originally proposed in 1967 by Veselago. ${ }^{1}$ Compelling experimental evidence for this behavior has been seen through negative index passbands, ${ }^{2-5}$ negative refraction, ${ }^{3,6}$ and apparent super-resolution imaging. ${ }^{7-10}$ These demonstrations have been made at room temperature in artificial loop-wire, ${ }^{2}$ dual circuit $^{11}$ and photonic crystal ${ }^{12}$ media.

An important prediction for NIR materials is evanescent wave amplification under the ideal condition of $n=-1+i 0$ precisely. ${ }^{14}$ This property will permit, in principle, image reconstruction with arbitrary precision and detail as demonstrated, for example, in calculations of flat-lens transfer functions. ${ }^{13-15}$ However, these theoretical works also show that ideal evanescent wave amplification suffers from three important constraints; the real part of $n$ must be -1 , exactly, the metamaterial must be thin (compared to the wavelength) to minimize retardation effects, and the imaginary part of $n$ must be much less than 1 , so that there is very little damping. The first two constraints can be satisfied, in principle, with appropriate engineering of existing metamaterials. However, significant losses have a debilitating effect on most designs.

The loss constraint is illustrated through calculations of the transfer function through a flat lens. The ppolarized light transfer function through a thin $\varepsilon_{\text {eff }}=-1+i$ $\varepsilon_{e f f}, \quad 2$ lens of thickness $d$ is approximately, ${ }^{14}$ $T_{p} \cong e^{-k_{x} d}\left(i \varepsilon_{\text {eff }, 2} / 2+e^{-2 k_{x} d}\right)^{-1}$, where $k_{x}$ is the lateral wavenumber. Exponential amplification requires $\varepsilon_{\text {eff, } 2}<<e^{-2 k_{x} d}$, which constrains both the upper spatial frequency that can be amplified and the slab thickness. For example, to amplify a lateral wavenumber with $k_{x} / k_{0}=$ 2 through a slab of thickness $d / \lambda=0.1$ requires $\varepsilon_{e f f, 2}<<8$ $\mathrm{x} 10^{-3}$. Such low losses are only available under special circumstances.

In this paper we consider the use of superconducting (SC) metals and low-loss dielectrics. These metamaterials reveal important properties, in addition to satisfying the third constraint mentioned above. We consider $\mathrm{Nb}$ wires (for negative effective permittivity, $\varepsilon_{e f f}$ ), and $\mathrm{Nb}$ split-ring resonators (SRRs, for negative effective permeability, $\left.\mu_{e f f}\right)$ in a quasi-one-dimensional waveguide geometry. ${ }^{4}$ The results are compared to predictions for the plasma edge of an infinite wire array ${ }^{16,17}$ and the magnetic properties of SRRs. ${ }^{18}$

A Ag-plated X-band (WR90) waveguide (interior dimensions $22.86 \mathrm{~mm}$ by $10.16 \mathrm{~mm}$ ) is employed for the cryogenic transmission measurements. The top and bottom walls of the 10-cm-long waveguide are perforated with $0.51-\mathrm{mm}$-diameter holes (5 holes wide, 21 rows long), and cylindrical 0.25 -mm-diameter $\mathrm{SC} \mathrm{Nb}$ wire is threaded through the holes to make a vertical wire array (Fig. 1, inset). Various periodic wire arrays can be created, including an $a=5.08 \mathrm{~mm}$ and a $45^{\circ}$-rotated $a=$ $7.19 \mathrm{~mm}$ square array. The $\mathrm{Nb}$ wire has a $\mathrm{SC}$ transition temperature $\left(\mathrm{T}_{\mathrm{c}}\right)$ of $9.25 \mathrm{~K}$, as measured by $\mathrm{AC}$ susceptibility.

The SRRs are made from 200-nm-thick Nb thin films RFmagnetron sputtered onto single crystal quartz substrates. The square SRRs ( $T_{c}$ of $8.65 \mathrm{~K}$ ) are photolithographically patterned and have a separation and gap width of $300 \mu \mathrm{m}$, a line width of $154 \mu \mathrm{m}$, an inner ring width of $1448 \mu \mathrm{m}$, and an outer ring width of $2360 \mu \mathrm{m}$, similar in design to Refs. [3,19]. The 350- $\mu$ m-thick quartz wafers are cut into chips $9.0 \mathrm{~mm}$ tall by $44.8 \mathrm{~mm}$ long, and placed into the waveguide to couple with the transverse magnetic fields of the microwave signals (Fig. 2, inset). The SRR gaps are oriented orthogonal to the electric field. In all cases, the SRR elements, wire diameters, and lattice spacing are all much smaller than the wavelength of the signals, creating an effective medium.

The waveguide assembly is placed in a magnetically shielded cryogenic Dewar and connected to an Agilent 8722D vector network analyzer through waveguide/coaxial couplers and nearly 1.37 meters of coaxial cable. The 2 by 2 complex S-matrix (including the transmission coefficient $S_{21}(\mathrm{f})$ ) of the waveguide is measured between $50 \mathrm{MHz}$ and $18 \mathrm{GHz}$. We compare these measurements to a waveguide wave matrix model, ${ }^{20}$ using effective medium models for the permittivity and permeability, and fit the data with a nonlinear leastsquares fit.

We first examined the plasma edge properties of a SC wire array with the dominant $\mathrm{TE}_{10}$ mode (electric field parallel to wires) for two different lattice parameters. Transmission data for the $a=7.19 \mathrm{~mm}$ lattice are shown in Fig. 1, at two temperatures, $7 \mathrm{~K}$ (below the wire $\mathrm{T}_{\mathrm{c}}$ ), 


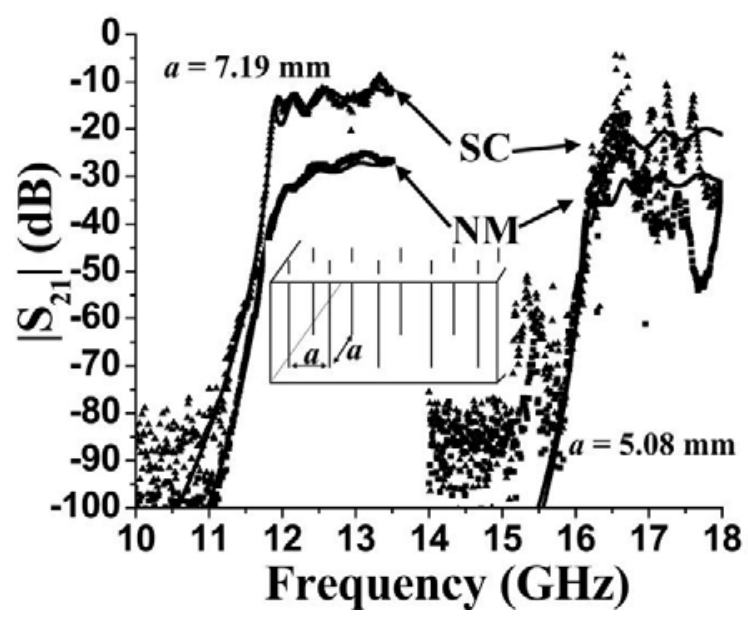

Fig. 1 Measurements of transmission magnitude $\left|S_{21}\right|$ versus frequency through $\mathrm{Nb}$ wire arrays in an X-band waveguide. Spectra on the left are for a lattice parameter $a=7.19 \mathrm{~mm}$, and those on the right are for $a=$ $5.08 \mathrm{~mm}$. The upper traces (triangles) are taken in the SC state at $7 \mathrm{~K}$, and the lower traces (squares) are taken in the NM state at room temperature. Theoretical fits to the data are solid lines. The inset shows a diagram of the wire array looking in the propagation direction.

and $297 \mathrm{~K}$.

It is readily seen that the insertion loss is reduced in the SC state, and the plasma edge is sharper. (All data presented here have been corrected for the insertion loss of the coaxial cables.) Fits using an effective dielectric response $^{16}$ of $\varepsilon_{\text {eff }}(\omega)=1-\omega_{p}^{2} / \omega(\omega+i \gamma)$ are also shown in Fig. 1. The loss parameters $(\gamma / 2 \pi)$ for the SC and normal metal (NM) states were found to be $24 \mathrm{MHz}$ and $146 \mathrm{MHz}$, respectively, illustrating the reduction of loss in the SC state. We find $\varepsilon_{\text {eff, } 2}=2.6 \times 10^{-3}(\mathrm{SC})$, and $\varepsilon_{\text {eff, } 2}=1.6 \times 10^{-2}(\mathrm{NM})$, below the plasma edge at 10.75 $\mathrm{GHz}$.

Pendry predicts the plasma edge of an infinite threedimensional cubic wire network to be given by ${ }^{16}$ $\omega_{p}^{2}=2 \pi c^{2}\left(a^{2}\left(\ln (a / r \sqrt{\pi})+\pi r^{2} / 2 a^{2}-1 / 2\right)\right)^{-1}$,

where $c$ is the speed of light, $a$ the lattice parameter, and $r$ the wire radius. Efros and Pokrovsky have a different prediction discussed in Ref. [17]. The fit plasma frequencies for this lattice in the SC (NM) state are $11.785 \mathrm{GHz}(11.728 \mathrm{GHz})$, which is somewhat larger than the predicted values of $9.64 \mathrm{GHz}^{16}$ and $9.95 \mathrm{GHz} .{ }^{17}$

Transmission data through the $a=5.08 \mathrm{~mm}$ lattice are also shown in Fig. 1 at $7 \mathrm{~K}$ and $291 \mathrm{~K}$. The plasma frequency increases as the wire density increases, as expected. This lattice has a fit plasma frequency of $16.135 \mathrm{GHz}(16.028 \mathrm{GHz})$ in the SC (NM) state, and predicted values of $14.52 \mathrm{GHz}^{16}$ and $16.9 \mathrm{GHz}{ }^{17}$ The results for this lattice are less clear because at least two higher order modes are excited in the waveguide above 15 $\mathrm{GHz}$. The model does not account for these modes, and so $\gamma / 2 \pi$ is found to be the same for both $(\sim 10 \mathrm{MHz})$, although the insertion loss decreases in the SC state. In

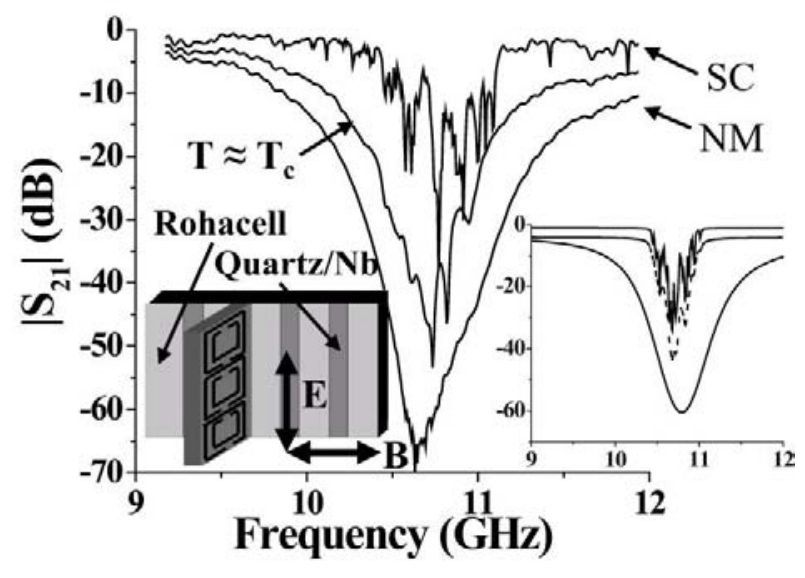

Fig. 2 Measurements of transmission magnitude $\left|S_{21}\right|$ versus frequency through a $\mathrm{Nb}$ /Quartz SRR array in an X-band waveguide. The upper trace is taken in the $\mathrm{SC}$ state at $5.0 \mathrm{~K}$, the middle trace is taken near $\mathrm{T}_{\mathrm{c}}$ of the SRRs, and the lower trace is taken in the NM state at $9.0 \mathrm{~K}$. The left inset shows a diagram of the SRR array looking in the propagation direction. The right inset shows theoretical models in the SC state (upper curve), close to $\mathrm{T}_{\mathrm{c}}$ (dashed), and NM state (lower curve).

all cases, no sudden changes in the transmission properties are seen at the wire $T_{c}$, and there is no evidence of enhanced transmission below a lower resonant frequency down to $50 \mathrm{MHz}$.

The results of the $\mathrm{Nb}$ /quartz $\mathrm{SRR}$ arrays in the waveguide are shown in Fig. 2. Four strips, two chips long (eighteen columns of three SRRs) were placed in the waveguide, spaced by Rohacell $31 \mathrm{HF}\left(\varepsilon_{r}=1.046\right.$ at 10 $\mathrm{GHz}$ ), as shown in the left inset. The SRRs are expected to resonate in the vicinity of $10.75 \mathrm{GHz}$, where one expects an $\varepsilon_{\text {eff }}>0, \mu_{\text {eff }}<0$ transmission notch. Note that in the NM state $(9.0 \mathrm{~K})$, the SRRs act collectively, giving a smooth notch centered at the expected frequency of $10.76 \mathrm{GHz}$. However, at $5.0 \mathrm{~K}$ (below $\mathrm{T}_{\mathrm{c}}$ ), the SRRs seem to act individually, and give a distribution of relatively high quality factor resonant frequencies, presumably determined by a distribution of local environments and interactions. ${ }^{21}$

These results are modeled well assuming that $\mu_{\text {eff }}^{18}$ takes on an average value given by the superposition, $\bar{\mu}_{e f f}=N^{-1} \sum_{1}^{N} \mu_{e f f}\left(f, f_{0 i}, \Gamma, F\right)$, where $f_{0 i}$ is the $i^{\text {th }}$ resonant frequency, $F$ and $\Gamma$ are the (assumed) global magnetic filling fraction and loss parameter. Based on the data, we take a Gaussian distribution (variable center frequency, and fixed width of $0.13 \mathrm{GHz}$ ) of $N=108$ resonant frequencies in the model. These model results are shown in the right inset of Fig. 2. Depending on the loss parameter, $\Gamma$, a smooth or jagged curve is obtained. The corresponding values of $\Gamma / 2 \pi$ for the SC state and the $\mathrm{NM}$ state are $13 \mathrm{MHz}$ and $637 \mathrm{MHz}$, respectively, and 48 $\mathrm{MHz}$ for the temperatures near $\mathrm{T}_{\mathrm{c}}$. Although this is 


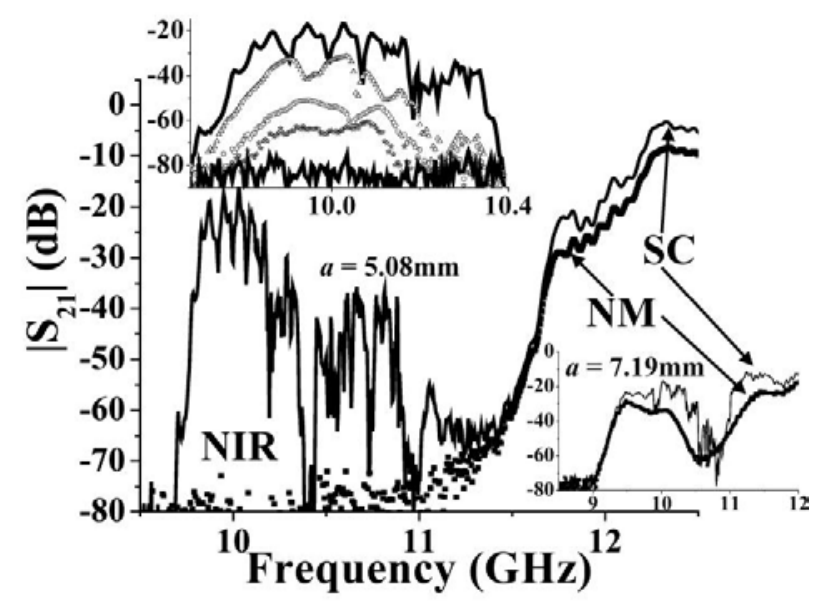

Fig. 3 Measurements of transmission magnitude $\left|S_{21}\right|$ versus frequency through a composite $90-\mathrm{mm}$-long $\mathrm{Nb}$ wire and $\mathrm{Nb}$ /Quartz SRR array in an X-band waveguide with $a=5.08 \mathrm{~mm}$. The upper trace (solid line) is taken in the fully SC state at $5.5 \mathrm{~K}$, and the lower trace (squares) is taken in the fully NM state at $11 \mathrm{~K}$. The left inset shows a close-up of the NIR region at $7.0,8.08,8.34,8.49$, and $8.77 \mathrm{~K}$, from top to bottom (SRR $\left.\mathrm{T}_{\mathrm{c}}=8.65 \mathrm{~K}\right)$. The right inset shows data from the composite with $a=$ $7.19 \mathrm{~mm}$ in the SC (solid line) and NM (squares) states.

an indirect way to determine $\mu_{\text {eff, } 2 \text {, estimates extracted }}$ from these calculations vary from $0.12 \leq \mu_{\text {eff, } 2} \leq 1.0$ (mean value 0.41 ) in the NM state, and $3.6 \times 10^{-4} \leq \mu_{\text {eff, } 2} \leq$ 0.4 (mean value 0.067 ) in the $\mathrm{SC}$ state.

Building upon these results, a SC metamaterial was created by combining a $\mathrm{Nb}$ wire array, with $\mathrm{Nb} \mathrm{SRR}$ strips supported by Rohacell in the middle of, and centers aligned with, the columns of wires. Fig. 3 contains the results of transmission measurements. The plasma edge of the $a=5.08 \mathrm{~mm}$ wire array has been significantly reduced by the presence of the $\mathrm{Nb} / \mathrm{Quartz} \mathrm{SRR}$ wafers, consistent with the results of Ref. [22]. In this case, the NIR peak is apparent in the SC state between 9.5 and 11 $\mathrm{GHz}$, consistent with the $\mu_{\text {eff }}<0$ notch frequency seen in the SRRs-only measurements (Fig. 2). Several observations support this interpretation. The left inset shows a close-up of the NIR peak as the temperature increases. As the temperature approaches the SRR film $\mathrm{T}_{\mathrm{c}}$, the peak becomes smooth, consistent with Fig. 2, and decreases in magnitude as the losses become significant. Most previous work has utilized thick $(\sim 30 \mu \mathrm{m}) \mathrm{Cu}$ plating, where $\Gamma / 2 \pi \sim 1 \mathrm{MHz}$, which is much less than our estimate $\left(\Gamma / 2 \pi \sim 1 \mathrm{GHz}^{18}\right)$ for the $\mathrm{Nb}$ thin film in the $\mathrm{NM}$ state just above $T_{c}$. It is clear that the high loss of the NM thin film SRRs prevents the observation of a NIR peak. In addition, the edge near $11.5 \mathrm{GHz}$ does not change at either the film or wire $T_{c}$, consistent with it being the plasma edge of the composite system.

To further support our picture, the right inset shows data for the $a=7.19 \mathrm{~mm}$ wire array and SRRs. It appears that the plasma edge is reduced to just above $9 \mathrm{GHz}$, and now the dip centered near $10.5 \mathrm{GHz}$ is due to the SRR resonance. That is, we have a $\mu_{\text {eff }}<0$ region above the plasma edge of the wires, and thus we do not have a NIR peak. In fact, below $T_{\mathrm{c}}$ the dip is jagged, and above $T_{\mathrm{c}}$ the dip is smooth, very similar to the $\varepsilon_{\text {eff }}>0, \mu_{\text {eff }}<0$ data in Fig. 2. From these results it is clear that the elements of the composite metamaterial are strongly interacting, and its properties are not simply a superposition of the wires and SRRs separately. ${ }^{22}$

We have created and examined the properties of a low-loss SC metamaterial. It displays sharp plasma edges with SC wires, and exhibits features in the SRR properties not seen in normal metals. Preliminary estimates show that $\varepsilon_{e f f, 2}$ and $\mu_{e f f, 2}$ have both been reduced by a factor of about 6, upon entering the SC state. We also demonstrate NIR behavior through superposition of $\varepsilon_{\text {eff }}<0, \mu_{\text {eff }}<0$ contributions in a SC composite metamaterial with a wire lattice parameter of $a=5.08 \mathrm{~mm}$. The present design shows substantial variation of the SRR resonant frequencies, even in a uniform array.

This work is supported by the National Science Foundation through grant NSF/ECS-0322844. We gratefully acknowledge assistance from R. Frizzell, J. Hamilton, K. Mercure, A. Prasad, and C. P. Vlahacos.

${ }^{1}$ V. G. Veselago, Usp. Fiz. Nauk 92, 517 (1967).

${ }^{2}$ D. R. Smith, W. J. Padilla, D. C. Vier, S. C. Nemat-Nasser, and S. Schultz, Phys. Rev. Lett. $\underline{84}, 4184$ (2000).

${ }^{3}$ R. A. Shelby, D. R. Smith, and S. Schultz, Science 292, 77 (2001). ${ }^{4}$ R. Marqués, J. Martel, F. Mesa, and F. Medina, Phys. Rev. Lett. $\underline{89}$, 183901 (2002), and I. G. Kondrat'ev and A. I. Smirnov, Phys. Rev. Lett. 91, 249401 (2003), and R. Marqués, J. Martel, F. Mesa, and F. Medina, Phys. Rev. Lett. 91, 249402 (2003).

${ }^{5}$ M. Bayindir, K. Aydin, E. Ozbay, P. Markos, and C. M. Soukoulis, Appl. Phys. Lett. 1 1, 120 (2002).

${ }^{6}$ A. A. Houck, J. B. Brock, I. L. Chuang, Phys. Rev. Lett. 90,137401 (2003).

${ }^{7}$ E. Cubukcu, K. Aydin, E. Ozbay, S. Foteinopolou1 and C.M. Soukoulis, Phys. Rev. Lett. 91, 207401 (2003).

${ }^{8}$ P. V. Parimi, W. T. Lu, P. Vodo, S. Sridhar, Nature 426, 404 (2003).

${ }^{9}$ A. N. Lagarkov and V. N. Kissel, Phys. Rev. Lett. 92, 077401 (2004).

${ }^{10}$ A. Grbic and G. V. Eleftheriades, Phys. Rev. Lett. 92, 117403 (2004).

${ }^{11}$ G. V. Eleftheriades, A. K. Iyer, and P. C. Kremer, IEEE Trans. Micro.

Th. Tech. $\underline{50}, 2702$ (2002).

${ }^{12}$ E. Cubukcu, K. Aydin, E. Ozbay, S. Foteinopolou1 and C.M.

Soukoulis, Nature $\underline{423}, 604$ (2003).

${ }^{13}$ J. B. Pendry, Phys. Rev. Lett. 85, 3966 (2000).

${ }^{14}$ J. T. Shen and P. M. Platzman, Appl. Phys. Lett. 80 , 3286 (2002).

${ }^{15}$ D. R. Smith, D. Schurig, M. Rosenbluth, S. Schultz, S. A.

Ramakrishna, J. B. Pendry, Appl Phys. Lett. 82, 1506 (2003).

${ }^{16}$ J. B. Pendry, A. J. Holden, W. J. Stewart, and I. Youngs, Phys. Rev. Lett. 76, 4773 (1996), and J. B. Pendry, A. J. Holden, D. J. Robbins, W. J. Stewart, J. Phys.: Condens. Matter, 10, 4785 (1998).

${ }^{17}$ A. L. Pokrovsky and A. L. Efros, Phys. Rev. Lett. 89, 093901 (2002).

${ }^{18}$ J. B. Pendry, A. J. Holden, D. J, Robbins, and W. J. Stewart, IEEE

Trans. Micro. Theo. Tech. 47, 2075 (1999).

${ }^{19}$ R. A. Shelby, D. R. Smith, S. C. Nemat-Nasser, and S. Schultz, Appl. Phys. Lett. 78,489 (2001).

${ }^{20}$ R. E. Collin, Field Theory of Guided Waves, (McGraw-Hill, New York, 1960).

${ }^{21}$ P. Gay-Balmaz, O. J. F. Martin, J. Appl. Phys. 92, 2929 (2002).

${ }^{22}$ T. Koschny, M. Kafesaki, E. N. Economou, C. M. Soukoulis, Phys. Rev. Lett. 93, 107402 (2004), and N. Katsarakis, T. Koschny, M. Kafesaki, E. N. Economou, E. Ozbay, C. M. Soukoulis, Phys. Rev. B $\underline{70}$, 201101 (2004). 